\title{
The reporting of outcomes in randomised controlled trials
}

\author{
THE SWITCH AND THE SPIN
}

\section{Ghert}

The British Editorial Society of Bone and Joint Surgery, London, United Kingdom
- M. Ghert, MD, FRCSC, Associate Professor of Surgery, McMaster University, 711 Concession Street Level B3 Surgical Offices Hamilton, ON, Canada.

Correspondence should be sent to M. Ghert; email: mghert@HHSC.ca

doi: 10.1302/2046-3758.610. BJR-2017-0296

Bone Joint Res 2017;6:600-601.
The randomised controlled trial (RCT) is considered the benchmark approach to determine the comparative efficacy of various clinical interventions. Rigorously designed RCTs are powered to detect a difference in a pre-defined primary outcome. Other secondary outcomes may be explored, but with the caveat that the study may not be powered to detect a difference between treatment groups for the secondary outcomes. Primary and secondary outcomes should be clearly specified and defined at the time of trial registration on ClinicalTrials.gov. This allows for accountability in reporting of the outcomes once the study is complete and the results are published.

It has become apparent in the surgical and orthopaedic literature that pre-registration of RCTs suffer from deficiencies, ${ }^{1}$ and that there are inconsistencies between the primary outcome registered and the primary outcome reported. ${ }^{2}$ This may occur because the findings for the primary outcome were not found to be statistically significant, and therefore the authors report any positive secondary outcome finding in lieu of reporting the primary outcome. This maneuvering of outcomes is termed 'spin' and is common in the medical literature. ${ }^{3}$ However, primary outcomes may indeed be negative ${ }^{4-7}$ and secondary outcomes may be positive, and can legitimately be reported as such.8,9 Transparent registering and reporting of outcomes, whether negative or positive, can alleviate the tendency to 'spin' results.

The candid reporting of outcomes in RCTs is imperative for various reasons. Outcomes may have an effect on patient management. ${ }^{10-12}$ In addition, reported outcomes can help design future RCTs, with and without patient involvement in protocol development. ${ }^{13,14}$ However, not all outcomes need to be considered primary or secondary. Smaller feasibility trials can be exploratory and report multiple predefined outcomes; ${ }^{15}$ however in these exploratory studies, the authors should not specify a primary outcome and should indicate that the trial is meant to generate hypotheses for further larger trials. Pilot RCTs are another form of RCT that do not report primary outcomes, but instead report feasibility metrics such as recruitment and protocol adherence..$^{16-18}$

However, in the case of adequately powered RCTs with a pre-defined primary outcome, it is the responsibility of the authors to report the primary outcome with minimal bias. A blinded and independent central adjudication committee can adjudicate all clinically significant outcomes, both primary and secondary, in order to maintain the integrity of the outcomes reporting and minimise bias in outcomes assessment. ${ }^{19}$ For these large RCTs, clear and transparent reporting of outcomes will allow the data reported to be included in meta-analyses, 20,21 which are the most likely study types to be considered in clinical practice guidelines and drive change in clinical practice.

Outcomes reporting in RCTs can be a complex and tricky business. Because orthopaedic surgeons are busy clinicians, often only the conclusions of an RCT will be quickly digested, and the methodology considered a less important read. However, a quick cross reference on ClinicalTrials.gov can determine that the primary outcome reported was predefined, and that the primary and secondary outcomes were not 'switched' in the reporting process. Such a rapid check as can instill confidence in the findings of the RCT and therefore improve translation of the findings into clinical practice when practice change is feasible and supported by biological rationale and patient preferences. 


\section{References}

1. Hannink G, Gooszen HG, Rovers MM. Comparison of registered and published primary outcomes in randomized clinical trials of surgical interventions. Ann Surg 2013;257:818-823.

2. Rongen JJ, Hannink G. Comparison of Registered and Published Primary Outcomes in Randomized Controlled Trials of Orthopaedic Surgical Interventions. J Bone Joint Surg [Am]2016;98-A:403-409.

3. Arunachalam L, Hunter IA, Killeen S. Reporting of Randomized Controlled Trials With Statistically Nonsignificant Primary Outcomes Published in High-impact Surgical Journals. Ann Surg 2017;265:1141-1145.

4. Waterson HB, Clement ND, Eyres KS, Mandalia VI, Toms AD. The early outcome of kinematic versus mechanical alignment in total knee arthroplasty: a prospective randomised control trial. Bone Joint J 2016;98-B:1360-1368.

5. van Tilburg CWJ, Stronks DL, Groeneweg JG, Huygen FJPM. Randomised sham-controlled double-blind multicentre clinical trial to ascertain the effect of percutaneous radiofrequency treatment for lumbar facet joint pain. Bone Joint $J$ 2016;98-B:1526-1533.

6. Huijbregts HJ, Khan RJ, Fick DP, et al. Component alignment and clinical outcome following total knee arthroplasty: a randomised controlled trial comparing an intramedullary alignment system with patient-specific instrumentation. Bone Joint J 2016;98-B:1043-1049.

7. Boonen B, Schotanus MGM, Kerens B, et al. No difference in clinical outcome between patient-matched positioning guides and conventional instrumented total knee arthroplasty two years post-operatively: a multicentre, double-blind, randomised controlled trial. Bone Joint J 2016;98-B:939-944.

8. Wyatt MC, Wright T, Locker J, et al. Femoral nerve infusion after primary total knee arthroplasty: a prospective, double-blind, randomised and placebo-controlled trial. Bone Joint Res 2015;4:11-16.

9. Yuenyongviwat $\mathbf{V}$, lamthanaporn $K$, Hongnaparak T, Tangtrakulwanich B. A randomised controlled trial comparing skin closure in total knee arthroplasty in the same knee: nylon sutures versus skin staples. Bone Joint Res 2016;5:185-190.

10. Dean BJF, Jones LD, Palmer AJR, et al. A review of current surgical practice in the operative treatment of proximal humeral fractures: does the PROFHER trial demonstrate a need for change? Bone Joint Res 2016;5:178-184.

11. Costa ML, Jameson SS, Reed MR. Do large pragmatic randomised trials change clinical practice?: assessing the impact of the Distal Radius Acute Fracture Fixation Trial (DRAFFT). Bone Joint J 2016;98-B:410-413.

12. Fullilove S, Gozzard C. Dorsally displaced fractures of the distal radius: a critical appraisal of the DRAFFT (distal radius acute fracture fixation trial) study. Bone Joint J 2016;98-B:298-300.

13. Handoll HHG, Brealey SD, Jefferson L, et al. Defining the fracture population in a pragmatic multicentre randomised controlled trial: PROFHER and the
Neer classification of proximal humeral fractures. Bone Joint Res 2016;5 481-489.

14. Huxley C, Achten J, Costa ML, Griffiths F, Griffin XL. A process evaluation of the WHiTE Two trial comparing total hip artroplasty with and without dual mobility component in the treatment of displaced intracapsular fractures of the proximal femur: can a trial investigating total hip arthroplasty for hip fracture be delivered in the NHS? Bone Joint Res 2016;5:444-452.

15. Karlakki SL, Hamad AK, Whittall C, et al. Incisional negative pressure wound therapy dressings (iNPWTd) in routine primary hip and knee arthroplasties: A randomised controlled trial. Bone Joint Res 2016;5:328-337.

16. Griffin XL, Parsons N, Achten J, Costa ML. A randomised feasibility study comparing total hip arthroplasty with and without dual mobility acetabular component in the treatment of displaced intracapsular fractures of the proximal femur : The Warwick Hip Trauma Evaluation Two : WHiTE Two. Bone Joint J. 2016;98-B:1431-1435.

17. PARITY Investigators. Prophylactic antibiotic regimens in tumour surgery (PARITY): a pilot multicentre randomised controlled trial. Bone Joint Res 2015;4:154-162.

18. Chesser TJS, Fox R, Harding $\mathbf{K}$, et al. The administration of intermittent parathyroid hormone affects functional recovery from trochanteric fractured neck of femur: a randomised prospective mixed method pilot study. Bone Joint J 2016;98B:840-845.

19. Nuttall J, Evaniew $\mathbf{N}$, Thornley $\mathbf{P}$, et al. The inter-rater reliability of the diagnosis of surgical site infection in the context of a clinical trial. Bone Joint Res. 2016;5: 347-352.

20. Sabharwal S, Patel NK, Griffiths D, et al. Trials based on specific fracture configuration and surgical procedures likely to be more relevant for decision making in the management of fractures of the proximal humerus: findings of a meta-analysis. Bone Joint Res 2016;5:470-480

21. Wilson DGG, Poole WEC, Chauhan SK, Rogers BA. Systematic review of aspirin for thromboprophylaxis in modern elective total hip and knee arthroplasty. Bone Joint J 2016:98-B:1056-1061.

\section{Funding Statement \\ None declared \\ Author Contribution \\ M. Ghert: Writing the paper. \\ Conflicts of Interest Statement \\ None declared}

() 2017 Ghert. This is an open-access article distributed under the terms of the Creative Commons Attributions licence (CC-BY-NC), which permits unrestricted use, distribution, and reproduction in any medium, but not for commercial gain, provided the original author and source are credited. 